Comment. Math. Helv. 72 (1997) 244-256

(C) 1997 Birkhäuser Verlag, Basel

$0010-2571 / 97 / 020244-13 \$ 1.50+0.20 / 0$

Commentarii Mathematici Helvetici

\title{
Partial resolutions and the zeta-function of a singularity
}

S. M. Gusein-Zade, I. Luengo and A. Melle-Hernández*

\begin{abstract}
We give a generalization of N. A'Campo's formula for the zeta-function of the monodromy of a singularity and show some examples of its applications.
\end{abstract}

Mathematics Subject Classification (1991). 14B05, 32S25, 32S45.

Keywords. Monodromy, zeta-function.

\section{Introduction}

Let $f:\left(\mathbb{C}^{n+1}, 0\right) \rightarrow(\mathbb{C}, 0)$ be a germ of a holomorphic function, $H=\{f=0\}$ (the germ of) its zero level hypersurface, $\chi_{f}$-the Euler characteristic of its Milnor fibre $V_{f}=\left\{z \in \mathbb{C}^{n+1}: f(z)=\epsilon,\|z\| \leq \delta\right\},(0<|\epsilon|<<\delta, \delta$ small enough $)$, $\zeta_{f}(t)$-the zeta-function of the (classical) monodromy, $\chi_{f}=\operatorname{deg} \zeta_{f}(t)$; for general notions see [2]. If the germ $f$ has an isolated critical point at the origin, then $\chi_{f}=1+(-1)^{n} \mu_{f}$, where $\mu_{f}$ is the Milnor number of the singularity $f$.

Let $\pi:(\mathcal{X}, \mathcal{D}) \rightarrow\left(\mathbb{C}^{n+1}, 0\right)$ be a modification of $\left(\mathbb{C}^{n+1}, 0\right)$, that is a proper analytic map of a non-singular $((n+1)$-dimensional $)$ manifold $\mathcal{X}$, which is an isomorphism over a dense open subset of a neighbourhood of the origin in $\mathbb{C}^{n+1}$, that is over the complement to the germ of a proper analytic subspace in $\left(\mathbb{C}^{n+1}, 0\right)$. If the (total) preimage $\pi^{-1}(H)$ of the zero level hypersurface $H$ is a divisor with normal crossings at each point of $\mathcal{X}$ and if $\pi$ is a modification of $H$ as well (i.e. if it is an isomorphism over a dense open subset of $H)$, then $\pi:(\mathcal{X}, \mathcal{D}) \rightarrow\left(\mathbb{C}^{n+1}, 0\right)$ is said to be a resolution of the singularity $f$ (or an embedded resolution of the hypersurface singularity $H=\{f=0\}$ ). Of course, in this case, $\mathcal{D}$ is a normal crossings divisor itself. The fact that the preimage $\pi^{-1}(H)$ is a divisor with normal crossings means that in a neighbourhood of any point of it, there exists a local system of coordinates $y_{0}, y_{1}, \ldots, y_{n}$ such that the lifting $\varphi=f \circ \pi$ of the function $f$ to the space $\mathcal{X}$ of the modification has the form $y_{0}^{m_{0}} \cdot y_{1}^{m_{1}} \cdot \ldots \cdot y_{n}^{m_{n}}$. Let $S_{m}$

*First author is partially supported by RFBR-95-01-01122a and INTAS-4373. Last two authors are partially supported by CAICYT PB91-0370-C02-01 and CAICYT PB94-291. 
be the set of points of the space $\mathcal{D}$, in a neighbourhood of which the lifting $\varphi$, in some local system of coordinates, has the form $y_{0}^{m}$ (in particular the intersections of the components of $\pi^{-1}(H)$ do not lie in this set), $\chi(A)$ will denote the Euler characteristic of the space $A$. The following result is due to N. A'Campo.

Theorem. [1]. Suppose that the modification $\pi:(\mathcal{X}, \mathcal{D}) \rightarrow\left(\mathbb{C}^{n+1}, 0\right)$ is a resolution of the singularity $f$ and that it is an isomorphism over the complement to the (germ of the) hypersurface $H=\{f=0\}$. Then

$$
\begin{gathered}
\chi_{f}=\sum_{m \geq 1} m \chi\left(S_{m}\right), \\
\zeta_{f}(t)=\prod_{m \geq 1}\left(1-t^{m}\right)^{\chi\left(S_{m}\right)} .
\end{gathered}
$$

Remark. The formula for the zeta-function differs by the minus sign in the exponent from that in [1] since we use the definition of the zeta-function from [2].

An important Remark. In $\S 1$ of [1], where the result is formulated (and thus in some other papers where it is cited), the requirement that the modification $\pi$ : $(\mathcal{X}, \mathcal{D}) \rightarrow\left(\mathbb{C}^{n+1}, 0\right)$ should be an isomorphism outside the zero-level hypersurface $H=\{f=0\}$ of the germ $f$ is not indicated explicitly. It is mentioned only in $\S 3$ in the proof of the Theorem. A similar remark about the theorem of A'Campo can be found e.g. in [8] (remark 2.4 and example 4.2).

The reason which makes the requirement essential is the following. Let $V_{\varphi}=$ $\{x \in \mathcal{X}: \varphi(x)=\epsilon\}$ be the non-singular level manifold of the lifting $\varphi=f \circ \pi$ of the function $f, \chi_{\varphi}=\chi\left(V_{\varphi}\right)$ its Euler characteristic, $\zeta_{\varphi}(t)$ - the zeta function of the monodromy of the function $\varphi$ (defined in the natural way). In fact the right-hand sides of the formulae $\left(\mathrm{A}^{\prime} \mathrm{C}\right)$ give the Euler characteristic of the level manifold and the zeta-function of the monodromy just for the function $\varphi$. They coincide with those for the singularity $f$ if the projection $\pi: V_{\varphi} \rightarrow V_{f}$ onto the Milnor fibre $V_{f}$ of $f$ is an isomorphism. In its turn, this projection is an isomorphism if and only if the modification $\pi:(\mathcal{X}, \mathcal{D}) \rightarrow\left(\mathbb{C}^{n+1}, 0\right)$ is an isomorphism outside the zero-level hypersurface $H$. Of course it is always possible to resolve a singularity $f$ by a modification which is an isomorphism outside $H=\{f=0\}$. However for applications, sometimes it is useful to consider resolutions which do not possess this property (see example 1 below).

The main object of the paper is to give a generalization of the theorem of $\mathrm{A}^{\prime}$ Campo for the case when the considered modification $\pi:(\mathcal{X}, \mathcal{D}) \rightarrow\left(\mathbb{C}^{n+1}, 0\right)$ is not a resolution of the singularity $f$. This generalization expresses the zeta-function of the singularity $f$ in terms of zeta-functions of the germs of the lifting $\varphi=f \circ \pi$ of the function $f$ at points of the exceptional divisor. It can be effectively applied 
in a situation when one can construct a partial resolution of a singularity $f$ with zeta-functions of all the germs of the lifting $\varphi=f \circ \pi$ known (e.g. obtained from the formula of A. Varchenko [9]).

We apply the obtained formula to singularities of the form $f=f_{d}+f_{d+k}+$ $\ldots\left(f_{m}\right.$ is homogeneous of degree $\left.m\right)$ with $f_{d}$ and $f_{d+k}$ satisfying some special conditions which has been extensively studied: E. Artal-Bartolo [3], I. Luengo[4], A. Melle-Hernández [5], D. Siersma [6], J. Stevens [7]. In particular we show how the formula of D. Siersma, [6] and J. Stevens, [7], for the zeta-function (or rather for the characteristic polynomial in their papers) of some isolated singularities of the form $f=f_{d}+f_{d+k}$ can be obtained in this way.

The authors are thankful to the referee of the paper for useful remarks and suggestions.

\section{Zeta-function via partial resolutions}

Let $\pi:(\mathcal{X}, \mathcal{D}) \rightarrow\left(\mathbb{C}^{n+1}, 0\right)$ be an arbitrary modification of $\left(\mathbb{C}^{n+1}, 0\right), f:\left(\mathbb{C}^{n+1}, 0\right)$ $\rightarrow(\mathbb{C}, 0)$ - a germ of an analytic function, $\varphi=f \circ \pi$ - its lifting to the space of the modification. At each point $x \in \pi^{-1}(H)$ the germ of the function $\varphi$ has a (non-isolated) singularity of a certain type. Let $\zeta_{\varphi, x}(t)$ be its zeta-function of the monodromy, $\chi_{\varphi, x}=\operatorname{deg} \zeta_{\varphi, x}(t)$ the Euler characteristic of its non-singular local level manifold. Let $\mathcal{S}=\{\Xi\}$ be a prestratification of $\mathcal{D}=\pi^{-1}(0)$ (that is a partitioning into semi-analytic subspaces without any regularity conditions) such that, for each stratum $\Xi$ of $\mathcal{S}$, the zeta-function $\zeta_{\varphi, x}(t)$ does not depend on $x$, for $x \in \Xi$. Let us denote this zeta-function by $\zeta_{\Xi}$ and by $\chi_{\Xi}$ its degree deg $\zeta_{\Xi}$. Let $\zeta_{\varphi}(t)$ be the zeta-function of the ("global") function $\varphi$ on $\mathcal{X}, \chi_{\varphi}=\operatorname{deg} \zeta_{\varphi}(t)$-the Euler characteristic of its non-singular level manifold $V_{\varphi}$.

\section{Theorem 1.}

$$
\begin{gathered}
\zeta_{\varphi}(t)=\prod_{\Xi \in \mathcal{S}}\left[\zeta_{\Xi}(t)\right]^{\chi(\Xi)}, \\
\chi_{\varphi}=\sum_{\Xi \in \mathcal{S}} \chi_{\Xi} \cdot \chi(\Xi) .
\end{gathered}
$$

If the modification $\pi:(\mathcal{X}, \mathcal{D}) \rightarrow\left(\mathbb{C}^{n+1}, 0\right)$ is an isomorphism outside the zerolevel hypersurface $H=\{f=0\}$, then the Milnor fibre $V_{\varphi}$, its Euler characteristic $\chi_{\varphi}$ and the zeta-function $\zeta_{\varphi}(t)$ of $\varphi$ coincide with those for $f: V_{f}=V_{\varphi}, \chi_{f}=$ $\chi_{\varphi}, \zeta_{f}(t)=\zeta_{\varphi}(t)$.

Corollary 1. If the modification $\pi:(\mathcal{X}, \mathcal{D}) \rightarrow\left(\mathbb{C}^{n+1}, 0\right)$ is an isomorphism outside $H=\{f=0\}$, then $\zeta_{f}(t)$ and $\chi_{f}$ are given by the same formulae (1) and (2). 
Proof of Theorem 1. First of all let us remark that the theorem of A'Campo obviously can be applied not only to a germ of an analytic function at a point, but also to a germ of an analytic function in a neighbourhood of a compact analytic subspace (such as the lifting $\varphi$ in a neighbourhood of $\mathcal{D}$ in $\mathcal{X}$ ). Let $\pi^{\prime}:\left(\mathcal{Y}, \mathcal{D}^{\prime}\right) \rightarrow$ $(\mathcal{X}, \mathcal{D})$ be a modification which is an isomorphism outside the zero-level set of $\varphi$ and which is a resolution of singularities of $\varphi$ (or equivalently $\pi \circ \pi^{\prime}:\left(\mathcal{Y}, \mathcal{D}^{\prime}\right) \rightarrow$ $\left(\mathbb{C}^{n+1}, 0\right)$ is a resolution of $\left.f\right)$. Because of the additivity of the Euler characteristic $(\chi(A \cup B)=\chi(A)+\chi(B)$ if $A \cap B=\emptyset)$ it is sufficient to prove the statement for any subpartition of $\mathcal{S}$. Let $\mathcal{S}^{\prime}$ and $\mathcal{S}^{*}$ be stratifications of $\mathcal{Y}$ and $\mathcal{X}$ respectively, such that:

(i) $\mathcal{S}^{\prime}$ and $\mathcal{S}^{*}$ are Whitney stratifications and the modification $\pi^{\prime}:\left(\mathcal{Y}, \mathcal{D}^{\prime}\right) \rightarrow$ $(\mathcal{X}, \mathcal{D})$ is a stratified morphism with respect to these stratifications.

(ii) $\mathcal{D}$ is a stratified subspace of the stratified space $\left(\mathcal{X}, \mathcal{S}^{*}\right)$.

(iii) For each stratum $\Xi^{\prime}$ of $\mathcal{S}^{\prime}$, the germs of the lifting $\varphi^{\prime}=\varphi \circ \pi^{\prime}$ at points of $\Xi^{\prime}$ have one and the same normal form (i.e. are equivalent to $y_{0}^{m_{0}} \cdot y_{1}^{m_{1}} \cdot \ldots \cdot y_{n}^{m_{n}}$ with one and the same set of powers $\left.m_{0}, m_{1}, \ldots, m_{n}\right)$.

The condition $(i)$ implies that the morphism $\pi^{\prime}$ is locally trivial over each stratum of $\mathcal{S}^{*}$. In particular if the stratum $\Xi$ of $\mathcal{S}^{*}$ is the image of the stratum $\Xi^{\prime}$ of $\mathcal{S}^{\prime}$, $\Xi=\pi^{\prime}\left(\Xi^{\prime}\right)$, then $\pi^{\prime}: \Xi^{\prime} \rightarrow \Xi$ is a smooth locally trivial fibre bundle. In particular

$$
\chi\left(\Xi^{\prime}\right)=\chi(\Xi) \cdot \chi\left(\left(\pi^{\prime}\right)^{-1}(x) \cap \Xi^{\prime}\right), \quad(x \in \Xi) .
$$

Let $S_{m}^{\prime}$ be the set of strata from $\mathcal{S}^{\prime}$ such that the germs of $\varphi^{\prime}$ at their points are equivalent to $y_{0}^{m} ; Y_{m}=\underset{\Xi^{\prime} \in \mathcal{S}^{\prime} m}{\cup} \Xi^{\prime}$. Using the theorem of A'Campo we have

$$
\begin{aligned}
& \zeta_{\varphi}=\prod_{m \geq 1}\left(1-t^{m}\right)^{\chi\left(Y_{m}\right)} \\
& =\prod_{m \geq 1}\left(1-t^{m}\right)^{\sum_{\Xi^{\prime} \in \mathcal{S}^{\prime} m}}{ }^{\chi\left(\Xi^{\prime}\right)}=\prod_{m \geq 1} \prod_{\Xi^{\prime} \in \mathcal{S}^{\prime} m}\left(1-t^{m}\right)^{\chi\left(\Xi^{\prime}\right)} \\
& =\prod_{m \geq 1} \prod_{\Xi \in \mathcal{S}} \prod_{\Xi^{\prime} \in S_{m}^{\prime} \cap\left(\pi^{\prime}\right)^{-1}(\Xi)}\left(1-t^{m}\right)^{\chi\left(\Xi^{\prime}\right)} \\
& =\prod_{\Xi \in \mathcal{S}} \prod_{m \geq 1} \prod_{\Xi^{\prime} \in S_{m}^{\prime} \cap\left(\pi^{\prime}\right)^{-1}(\Xi)}\left(1-t^{m}\right)^{\chi(\Xi) \cdot \chi\left(\left(\pi^{\prime}\right)^{-1}(x) \cap \Xi^{\prime}\right)} \\
& =\prod_{\Xi \in \mathcal{S}}\left(\prod_{m \geq 1} \prod_{\Xi^{\prime} \in S_{m}^{\prime} \cap\left(\pi^{\prime}\right)^{-1}(\Xi)}\left(1-t^{m}\right)^{\chi\left(\left(\pi^{\prime}\right)^{-1}(x) \cap \Xi^{\prime}\right)}\right)^{\chi(\Xi)}=\prod_{\Xi \in \mathcal{S}}\left[\zeta_{\Xi}(t)\right]^{\chi(\Xi)}
\end{aligned}
$$

The strata outside $\mathcal{D}$ can be excluded from the formula since their Euler characteristics are equal to zero. As usual the formula for the Euler characteristic of the Milnor fibre follows from the formula for the zeta-function. 
For some applications of Theorem 1 we need to have a possibility to treat cases when the modification under consideration is not an isomorphism outside the zerolevel hypersurface $H=\{f=0\}$ of the germ $f$. Here we do not try to formulate a general statement, but describe a situation which we use in examples below.

Let $\mathbb{C}^{n+1}$ be represented as the product $\mathbb{C}^{n+1}=\mathbb{C}^{n} \times \mathbb{C}^{1}, f:\left(\mathbb{C}^{n+1}, 0\right) \rightarrow(\mathbb{C}, 0)$ a germ of an analytic function such that the restriction of $f$ to $\mathbb{C}^{1}=\{0\} \times \mathbb{C}^{1}$ is not equal to zero. Let $d$ be the multiplicity of this restriction at the origin in $\mathbb{C}^{1}$. Let $\pi:(\mathcal{X}, \mathcal{D}) \rightarrow\left(\mathbb{C}^{n}, 0\right)$ be a modification which is an isomorphism outside the origin in $\mathbb{C}^{n}, \tilde{\pi}=\pi \times i d:\left(\mathcal{X} \times \mathbb{C}^{1}, \mathcal{D} \times 0\right) \rightarrow\left(\mathbb{C}^{n} \times \mathbb{C}^{1}, 0 \times 0\right)$ the corresponding modification of $\mathbb{C}^{n+1}=\mathbb{C}^{n} \times \mathbb{C}^{1}$ ("the trivial extension"), $\varphi=f \circ \tilde{\pi}:\left(\mathcal{X} \times \mathbb{C}^{1}, \mathcal{D} \times 0\right) \rightarrow(\mathbb{C}, 0)$.

Theorem 2.

$$
\begin{aligned}
\zeta_{\varphi}(t) & =\zeta_{f}(t) \cdot\left(1-t^{d}\right)^{\chi(\mathcal{D})-1} \\
\chi_{\varphi} & =\chi_{f}+d \cdot(\chi(\mathcal{D})-1) .
\end{aligned}
$$

Proof. Let $\Gamma_{f}: V_{f} \rightarrow V_{f}$ be the classical monodromy transformation of the singularity $f, \Gamma_{\varphi}: V_{\varphi} \rightarrow V_{\varphi}$-that of the function $\varphi$. We can suppose that (the diffeomorphism) $\Gamma_{f}$ preserves the intersection of $V_{f}$ with the line $\mathbb{C}^{1}$. The intersection $V_{f} \cap \mathbb{C}^{1}$ consists of $d$ points, $\Gamma_{f}$ is a cyclic permutation of them. The zeta-function of the cyclic permutation of $d$ points is equal to $\left(1-t^{d}\right)$. The projection $\tilde{\pi}: V_{\varphi} \rightarrow V_{f}$ is an isomorphism outside $V_{f} \cap \mathbb{C}^{1}$, the preimage of each point from $V_{f} \cap \mathbb{C}^{1}$ coincides with $\mathcal{D}$. The transformation $\Gamma_{\varphi}$ permutes $d$ copies of $\mathcal{D}$ cyclically. The zeta-function of such transformation of $\mathcal{D} \times\{d$ points $\}$ is equal to $\left(1-t^{d}\right)^{\chi(\mathcal{D})}$. The result follows from the multiplication property of the zeta-function of a transformation, (see [2] p. 94).

\section{Applications and examples}

Let $f:\left(\mathbb{C}^{n+1}, 0\right) \rightarrow(\mathbb{C}, 0)$ be a germ of a holomorphic function, $f=f_{d}+f_{d+k}+\ldots$ -its decomposition into the sum of its homogeneous parts $\left(\operatorname{deg} f_{m}=m, f_{d} \neq 0\right.$ ). For a homogeneous $f_{m}$, let $\mathcal{Z}\left(f_{m}\right)$ be the hypersurface in $\mathbb{C P}^{n}$ defined by the equation $\left\{f_{m}=0\right\}, \mathcal{Z}_{\text {red }}\left(f_{m}\right)$ the reduced hypersurface, $S\left(f_{m}\right)=S\left(\mathcal{Z}\left(f_{m}\right)\right)$-the singular locus of $\mathcal{Z}\left(f_{m}\right)$.

3.1. It is possible to start a resolution of $f$ with the blowing-up at the origin. While treating a singularity this way, one meets the problem to calculate the zetafunction of the singularity $\varphi:\left(\mathbb{C}^{n+1}, 0\right) \rightarrow(\mathbb{C}, 0)$ of the form $\varphi\left(u, y_{1}, \ldots, y_{n}\right)=$ $u^{d}\left(g\left(y_{1}, \ldots, y_{n}\right)+u^{k}\right)$, where $g:\left(\mathbb{C}^{n}, 0\right) \rightarrow(\mathbb{C}, 0)$ is an analytic germ. Let us analyse the case when $g$ has an isolated critical point at the origin.

Let $\pi^{\prime}:(\mathcal{Y}, \mathcal{D}) \rightarrow\left(\mathbb{C}^{n}, 0\right)$ be a resolution of the singularity $g$ which is an isomorphism outside the origin. For $\bar{m}=\left(m_{1}, m_{2}, \ldots, m_{n}\right)$ with integer $m_{1} \geq$ 
$m_{2} \geq \ldots \geq m_{n} \geq 0$, let $S_{\bar{m}}$ be the set of points of $\mathcal{D}$ at which the lifting of $g$ has the normal form $y_{1}^{m_{1}} \cdot \ldots \cdot y_{n}^{m_{n}}, S_{m}=S_{(m, 0, \ldots, 0)}$. Let us take the trivial extension $\pi^{\prime} \times i d:\left(\mathcal{Y} \times \mathbb{C}^{1}, \mathcal{D} \times 0\right) \rightarrow\left(\mathbb{C}^{n} \times \mathbb{C}^{1}, 0 \times 0\right)$ of this modification, let $\psi$ be the lifting of $\varphi$. At a point of $S_{\bar{m}}$ the germ of $\psi$ has the normal form $u^{d} \cdot\left(y_{1}^{m_{1}} \cdot \ldots \cdot y_{n}^{m_{n}}+u^{k}\right)$. The zeta-function of such a germ can be obtained by the formula of Varchenko [9]. If there are more than one power $m_{i}$ different from zero, then it is equal to $\left(1-t^{d+k}\right)$. At a point of $S_{m}$ the local zeta-function of $\psi$ is equal to

$$
\left(1-t^{d+k}\right)\left(1-t^{\left(\frac{m}{m^{\prime}}\right)(d+k)}\right)^{-m^{\prime}}
$$

where $m^{\prime}=$ g.c.d. $(m, k)$. By the formula of A'Campo

$$
\zeta_{g}(t)=\prod\left(1-t^{m}\right)^{\chi\left(S_{m}\right)}
$$

According to Theorems 1 and 2

$$
\begin{aligned}
\zeta_{\varphi}(t) & =\left(1-t^{d+k}\right)^{1-\chi(\mathcal{D})} \\
& \cdot \prod_{\bar{m} \neq(m, 0, \ldots, 0)}\left(1-t^{d+k}\right)^{\chi\left(S_{\bar{m}}\right)} \prod_{m \geq 1}\left(\left(1-t^{d+k}\right)\left(1-t^{\left(\frac{m}{m^{\prime}}\right)(d+k)}\right)^{-m^{\prime}}\right)^{\chi\left(S_{m}\right)} \\
& =\left(1-t^{d+k}\right) \prod_{m \geq 1}\left(1-t^{\left(\frac{m}{m^{\prime}}\right)(d+k)}\right)^{-m^{\prime} \cdot \chi\left(S_{m}\right)}
\end{aligned}
$$

since $\sum_{\bar{m}} \chi\left(S_{\bar{m}}\right)=\chi(\mathcal{D})$.

Zeta-function is defined for any map $h: U \rightarrow U$ of a space $U$ into itself (see [2]). Let us denote the zeta-function of the power $h^{k}$ of the map $h$ by $\zeta_{h}^{k}(t)($ $\left.\zeta_{h}(t)=\zeta_{h}^{1}(t)\right)$. The zeta-function $\zeta_{h}^{k}(t)$ is determined by the zeta-function $\zeta_{h}(t)$ of the map $h$ itself in the obvious way. In particular, if $\zeta_{h}(t)=\prod_{m \geq 1}\left(1-t^{m}\right)^{a_{m}}$, then

$$
\zeta_{h}^{k}(t)=\prod_{m \geq 1}\left(1-t^{\frac{m}{g . c . d .(k, m)}}\right)^{g . c . d .(k, m) \cdot a_{m}} .
$$

For a germ $g$ of a function, let $\zeta_{g}^{k}(t)$ be the zeta-function $\zeta_{\Gamma}^{k}(t)$ for the classical monodromy transformation $\Gamma=\Gamma_{g}$ of $g$. Thus we have proved

\section{Lemma 1.}

$$
\zeta_{\varphi}(t)=\left(1-t^{d+k}\right)\left(\zeta_{g}^{k}\left(t^{d+k}\right)\right)^{-1}
$$

3.2. Let $f=f_{d}+f_{d+k}+\ldots$ and let us suppose that $S\left(f_{d}\right)$ is finite (consists of $s$ points $\left.P_{1}, \ldots, P_{s}\right), f_{d+k}\left(P_{i}\right) \neq 0$ (i.e. $\mathcal{Z}\left(f_{d+k}\right) \cap S\left(f_{d}\right)=\emptyset$ ). Let us blow-up 
the origin in $\mathbb{C}^{n+1}$. Let $\varphi$ be the lifting of $f$ to the space $\mathcal{X}$ of the modification. Let us consider the partitioning (in fact a stratification) of the exceptional divisor $E \simeq \mathbb{C P}^{n}$ of this blowing-up into the following strata

(a) 0 -dimensional strata $\Xi_{i}^{0},(i=1, \ldots, s)$ each consisting of one point $P_{i}$;

(b) $(n-1)$-dimensional stratum $\Xi^{n-1}=\mathcal{Z}\left(f_{d}\right) \backslash\left\{P_{1}, \ldots, P_{s}\right\}$;

(c) $n$-dimensional stratum $\Xi^{n}=\mathbb{C P}^{n} \backslash \mathcal{Z}\left(f_{d}\right)$.

In a neighbourhood of the point $P_{i}$ in $\mathcal{X}$, there exists a coordinate system $u, y_{1}, \ldots, y_{n}$ such that (locally) $\mathbb{C P}^{n}$ coincides with the hyperplane $\{u=0\}$ and the lifting $\varphi$ has the form $\varphi=\varphi_{i}=v \cdot u^{d} \cdot\left(g_{i}\left(y_{1}, \ldots, y_{n}\right)+u^{k}\right)$, where $v$ is invertible $(v(0) \neq 0)$ (further we will simply omit such "nonessential" factors), $g_{i}:\left(\mathbb{C}^{n}, 0\right) \rightarrow(\mathbb{C}, 0)$ is a germ of an analytic function (with an isolated critical point at the origin) the zero level locus of which coincides with the germ of the hypersurface $\mathcal{Z}\left(f_{d}\right)$ at $P_{i}$. Let $\mu_{i}$ be the Milnor number of $g_{i}$.

The Euler characteristic of the stratum $\Xi^{n-1}$ is equal to $\chi(n, d)-\sum_{i=1}^{s}\left((-1)^{n-1} \mu_{i}+1\right)$, where $\chi(n, d)=(n+1)+\frac{(1-d)^{n+1}-1}{d}$ is the Euler characteristic of a non-singular hypersurface of degree $d$ in $\mathbb{C P}^{n}$. At each point of $\Xi^{n-1}$ the lifting $\varphi$ has the normal form $u^{d} \cdot y_{1}$. Its zeta-function is equal to 1 .

The Euler characteristic of the stratum $\Xi^{n}$ is equal to

$$
\chi\left(\mathbb{C P}^{n}\right)-\chi\left(\mathcal{Z}\left(f_{d}\right)\right)=(n+1)-\chi(n, d)+(-1)^{n-1} \sum_{i=1}^{s} \mu_{i} .
$$

At each point of $\Xi^{n}$ the lifting $\varphi$ has the normal form $u^{d}$. Its zeta-function is equal to $\left(1-t^{d}\right)$.

Theorem 1 (or rather the Corollary 1) gives

$$
\zeta_{f}(t)=\left(1-t^{d}\right)^{\chi\left(\Xi^{n}\right)} \prod_{i=1}^{s} \zeta_{\varphi_{i}}(t) .
$$

Using Lemma 1 we have

$$
\zeta_{f}(t)=\left(1-t^{d}\right)^{\chi\left(\Xi^{n}\right)}\left(1-t^{d+k}\right)^{s}\left(\prod_{i=1}^{s} \zeta_{g_{i}}^{k}\left(t^{d+k}\right)\right)^{-1} .
$$

Let $h$ be a homogeneous polynomial of degree $d$ in $(n+1)$ variables such that $\mathcal{Z}(h)$ is non-singular. Then the function $h$ has an isolated critical point at the origin. The zeta-function of it is equal to

$$
\zeta_{d, n}(t)=\left(1-t^{d}\right)^{(n+1)-\chi(n, d)}
$$

Thus

$$
\zeta_{f}(t)=\zeta_{d, n}(t)\left(1-t^{d}\right)^{(-1)^{n-1} \sum_{i=1}^{s} \mu_{i}}\left(1-t^{d+k}\right)^{s}\left(\prod_{i=1}^{s} \zeta_{g_{i}}^{k}\left(t^{d+k}\right)\right)^{-1} .
$$


This is just the formula of J. Stevens and D. Siersma in terms of the zeta-function.

3.3. Let us suppose that $f=f_{d}+f_{d+k}$ and that:

(i) the singular locus $S\left(f_{d}\right)$ is one-dimensional (i.e. it consists of several points and a projective curve $C$ );

(ii) the curve $\mathrm{C}$ is reduced;

(iii) $\mathcal{Z}\left(f_{d+k}\right)$ intersects $S\left(f_{d}\right)$ only at non-singular points of $C$, at each point of intersection $\mathcal{Z}_{\text {red }}\left(f_{d+k}\right)$ is smooth itself and intersects $C$ transversally.

The condition $(i i)$ means that the zero locus $\mathcal{Z}\left(f_{d}\right)$ has the transversal singularity type $A_{1}$ at a generic point of $C$. Let $\tilde{C}$ be the set of (non-singular) points of $C$ with the transversal singularity type $A_{1}, S\left(f_{d}\right)=\tilde{C} \cup\left\{P_{1}, \ldots, P_{s}\right\}$ with different points $P_{1}, \ldots, P_{s}$. Let $\pi:\left(\mathcal{X}, \mathbb{C P}^{n}\right) \rightarrow\left(\mathbb{C}^{n+1}, 0\right)$ be the blowing-up of the origin in $\mathbb{C}^{n+1}, \varphi$ the lifting of $f$ to the space $\mathcal{X}$. Let us consider the partitioning of the exceptional divisor $\mathbb{C P}^{n}$ into the following strata

(a) 0-dimensional strata $\Xi_{i}^{0}$ consisting of one point $P_{i}$ each $(i=1, \ldots, s)$;

(b) 0-dimensional strata $\Lambda_{j}^{0},(j=1, \ldots, r)$ each consisting of one point of intersection of $\mathcal{Z}\left(f_{d+k}\right)$ with $S\left(f_{d}\right)$ (except those which coincide with one of $P_{i}$ );

(c) 1-dimensional stratum $\Xi^{1}=\tilde{C}$;

(d) $(n-1)$-dimensional stratum $\Xi^{n-1}=\mathcal{Z}\left(f_{d}\right) \backslash S\left(f_{d}\right)$;

(e) $n$-dimensional stratum $\Xi^{n}=\mathbb{C P}^{n} \backslash \mathcal{Z}\left(f_{d}\right)$.

At each point of $\Xi^{n}$, the lifting $\varphi$ of the germ $f$ has the normal form $u^{d}$. Its zeta-function is equal to $\left(1-t^{d}\right)$.

At each point of $\Xi^{n-1}$, the lifting $\varphi$ has the normal form $u^{d} \cdot y_{1}$. Its zeta-function is equal to 1 .

At a point of $\Xi^{1}$, the lifting $\varphi$ has the normal form $u^{d}\left(y_{1}^{2}+\ldots+y_{n-1}^{2}+u^{k}\right)$. The zeta-function of it can be obtained by the formula of Varchenko. But it is easier to get it from Lemma 1 (applied to the function $\left.g\left(y_{1}, \ldots, y_{n-1}\right)=y_{1}^{2}+\ldots+y_{n-1}^{2}\right)$. One has

$$
\zeta_{g}(t)=\left(1-t^{2}\right)^{(n-1)-\chi(n-2,2)}= \begin{cases}1, & \text { if } n \text { is odd, } \\ \left(1-t^{2}\right), & \text { if } n \text { is even. }\end{cases}
$$

Hence, for a point of $\Xi^{1}$, the local zeta-function of $\varphi$ is equal to

$$
\zeta^{(n, k)}(t)= \begin{cases}\left(1-t^{d+k}\right), & \text { if } n \text { is odd, } \\ \left(1-t^{d+k}\right)\left(1-t^{2(d+k)}\right)^{-1}, & \text { if } n \text { is even and } k \text { is odd } \\ \left(1-t^{d+k}\right)^{-1}, & \text { if both } n \text { and } k \text { are even. }\end{cases}
$$

At a point $\Lambda_{j}^{0}$, the normal form of $\varphi$ is $u^{d}\left(y_{1}^{2}+\ldots+y_{n-1}^{2}+y_{n}^{q} u^{k}\right)$. Its zeta-function is equal to $1([9])$.

At a point $P_{i}$, the lifting $\varphi$ has the form $\varphi_{i}=u^{d}\left(g_{i}\left(y_{1}, \ldots, y_{n-1}\right)+u^{k}\right)$, where $\left\{g_{i}=0\right\}$ is the local equation of the zero locus $\mathcal{Z}\left(f_{d}\right)$ at the point $P_{i}$. Its zetafunction is equal to

$$
\left(1-t^{d+k}\right)\left(\zeta_{g_{i}}^{k}\left(t^{d+k}\right)\right)^{-1}
$$


Remark 1. In principle we can not apply Lemma 1 to this case directly since the singularity $g_{i}$ might be non-isolated (if $P_{i} \in C$ ). In our case it means that a resolution of the singularity $g_{i}$ demands some modifications outside the origin. However, it is possible to show that the statement is valid for this case as well . The reasoning is as follows. There exists a modification (a partial resolution of the singularity $g_{i}$ ) which is an isomorphism outside the origin and which resolves all the singularities of $g_{i}$ except those on the branches of $S\left(f_{d}\right)$. We can also suppose that it resolves the singularity of the curve $S\left(f_{d}\right)$ itself. It means that the strict transform of $S\left(f_{d}\right)$ consists of several nonsingular branches which intersect the exceptional divisor transversally at its non singular points. At such a point the lifting of $g_{i}$ to the space of the modification has the normal form $y_{1}\left(y_{2}^{2}+\ldots+y_{n-1}^{2}\right)$ and the lifting of $\varphi_{i}$ to the trivial extension of this modification has the form $u^{d}\left(y_{1}\left(y_{2}^{2}+\ldots+y_{n-1}^{2}\right)+u^{k}\right)$. That is, they are different from those which can be met for a (complete) resolution. However the zeta-function of $y_{1}\left(y_{2}^{2}+\ldots+y_{n-1}^{2}\right)$ is equal to 1 , and that of $u^{d}\left(y_{1}\left(y_{2}^{2}+\ldots+y_{n-1}^{2}\right)+u^{k}\right)$ equals to $\left(1-t^{d+k}\right)$. So, they exactly coincide with the zeta-functions corresponding to strata $S_{\bar{m}},\left(\bar{m}=\left(m_{1}, \ldots, m_{n}\right)\right)$ of a resolution with more than one $m_{i}$ different from zero. Therefore, the proof goes in the same way.

Thus we have

$$
\zeta_{f}(t)=\left(1-t^{d}\right)^{\chi\left(\mathbb{C P}^{n} \backslash \mathcal{Z}\left(f_{d}\right)\right)} \cdot\left(\zeta^{(n, k)}(t)\right)^{\chi\left(\Xi^{1}\right)}\left(1-t^{d+k}\right)^{s}\left(\prod_{i=1}^{s} \zeta_{g_{i}}^{k}\left(t^{d+k}\right)\right)^{-1}
$$

3.4. Let $n+1$ be equal to 3 . Let $f_{10}$ be the homogeneous form $f_{10}=\left(x^{2} z^{3}+y^{5}\right)^{2}$. The curve $C=\mathcal{Z}\left(x^{2} z^{3}+y^{5}\right)$ has two singular points $P_{1}=(0: 0: 1)$ and $P_{0}=(1: 0: 0)$ of the type $A_{4}$ and $E_{8}$ respectively. The Euler characteristic of $C$ is equal to 2 . Let us consider plane curves defined by homogeneous forms $h_{11}$ of degree 11 . The space of these forms such that $\mathcal{Z}\left(h_{11}\right)$ is smooth at $P_{1}$ and the intersection number of $\mathcal{Z}\left(f_{11}\right)$ and $C$ at $P_{1}$ is equal to 5 has dimension 74 . So we can take one of them, $f_{11}$, with the condition that all remaining intersections of $\mathcal{Z}\left(f_{11}\right)$ and $C$ are transversal (so there are 50 of them, $\left.P_{2}, \ldots, P_{51}\right)$.

Remark 2. In this dimension, the singularities of $f=f_{d}+f_{d+k}$ such that the set $S\left(\mathcal{Z}\left(f_{d}\right)\right) \cap S\left(\mathcal{Z}\left(f_{d+k}\right)\right)$ is empty have been studied in [5], they are isolated and their Milnor number is equal to

$$
(d-1)^{3}+k \cdot\left(\chi\left(\mathcal{Z}\left(f_{d}\right)\right)-3 d+d^{2}+\sum_{P}\left(I_{p}\left(\mathcal{Z}\left(f_{d}\right), \mathcal{Z}\left(f_{d+k}\right)\right)-1\right)\right),
$$

where in the sum $P \in S\left(\mathcal{Z}\left(f_{d}\right)\right) \cap \mathcal{Z}\left(f_{d+k}\right)$ and $I_{p}\left(\mathcal{Z}\left(f_{d}\right), \mathcal{Z}\left(f_{d+k}\right)\right)$ denotes the intersection multiplicity of these curves at $P$. So $f=f_{10}+f_{11}$ is isolated and its Milnor number is equal to 860 . 
After blowing-up the origin, we have the following natural stratification of the exceptional divisor $\mathbb{C P}^{2}$

(a) 0-dimensional stratum consisting of one point $P_{0}$;

(b) 0-dimensional stratum consisting of one point $P_{1}$;

(c) 0-dimensional stratum $\Xi^{0}$ consisting of 50 points, $\left\{P_{2}, \ldots, P_{51}\right\}$;

(d) 1-dimensional stratum $\Xi^{1}=C \backslash\left\{P_{i}\right\}$;

(e) 2-dimensional stratum $\Xi^{2}=\mathbb{C P}^{2} \backslash C$.

At each point of $\Xi^{2}$, the lifting of the germ $f$ has the normal form $u^{10}$. Its zeta-function is equal to $\left(1-t^{10}\right)$ and $\chi\left(\Xi^{2}\right)=\chi\left(\mathbb{C P}^{2}\right)-\chi(C)=1$.

At each point of $\Xi^{1}$, the lifting of $f$ has the normal form $u^{10}\left(x^{2}+u\right)$. Its zeta-function is equal to $\left(1-t^{11}\right)^{-1}, \chi\left(\Xi^{1}\right)$ is equal to $(-50)$.

At each point of $\Xi^{0}$, the lifting of $f$ has the normal form $u^{10}\left(x^{2}+y u\right)$. Its zeta-function is equal to 1 .

At the point $P_{0}$, the lifting of $f$ has the normal form $\varphi_{0}=u^{10}\left(\left(z^{3}+y^{5}\right)^{2}+u\right)$. The zeta-function of this germ is equal to

$$
\left(1-t^{11}\right)\left(\zeta_{g}\left(t^{11}\right)\right)^{-1}
$$

where $\zeta_{g}(t)$ is the zeta-function of the germ $g=\left(z^{3}+y^{5}\right)^{2}$ (equal to $\frac{\left(1-t^{6}\right)\left(1-t^{10}\right)}{\left(1-t^{30}\right)}$ ). Thus

$$
\zeta_{\varphi_{0}}=\left(1-t^{11}\right) \frac{\left(1-t^{330}\right)}{\left(1-t^{66}\right)\left(1-t^{110}\right)} \text {. }
$$

Remark 3. In fact by blowing-up the origin one reduces the problem of calculating the zeta-function of any singularity of the form $f_{d}+f_{d+k}$ (i.e. the sum of two homogeneous polynomials), isolated or not, to the problem of calculating zeta-functions of germs of the form $u^{d}\left(g(x, y)+h(x, y) \cdot u^{k}\right)$.

At the point $P_{1}$, the lifting of $f$ has the normal form $\varphi_{1}=u^{10}\left(\left(z^{3}+y^{5}\right)^{2}+x u\right)$. The singularity $\varphi_{1}$ is not non-degenerate with respect to its Newton diagram, so we cannot apply the formula of A. Varchenko directly. In order to use the formula of N. A'Campo we have to construct an embedded resolution of a surface singularity, what is more complicated than for a plane curve singularity. It is possible to calculate the zeta-function of such germ by using the following procedure.

We make an embedded resolution $\pi:(\mathcal{X}, \mathcal{D}) \rightarrow\left(\mathbb{C}^{2}, 0\right)$ of the curve singularity of $\{g=0\} \cup\{h=0\}$ (or equivalently a resolution of the product germ $g h$ ), which is an isomorphism outside the origin. At each point of the exceptional divisor of the resolution we have local forms $y_{1}^{m_{1}} y_{2}^{m_{2}}$ and $y_{1}^{l_{1}} y_{2}^{l_{2}}$ for the liftings of the germs $g$ and $h$ respectively. Let us take the trivial extension $\pi:\left(\mathcal{X} \times \mathbb{C}^{1}, \mathcal{D} \times 0\right) \rightarrow\left(\mathbb{C}^{2} \times \mathbb{C}^{1}, 0\right)$ of this modification. It is not an isomorphism over the line $0 \times \mathbb{C}^{1}$, which belongs to the zero locus of the germ $\varphi_{1}$. Thus the zeta-functions of $\varphi_{1}$ and of its lifting coincide. At the corresponding point the lifting of the germ $\varphi_{1}$ to the space of this modification has the normal form $u^{d}\left(y_{1}^{m_{1}} y_{2}^{m_{2}}+u^{k} y_{1}^{l_{1}} y_{2}^{l_{2}}\right)$. Its zeta-function is given by the formula of A. Varchenko. 


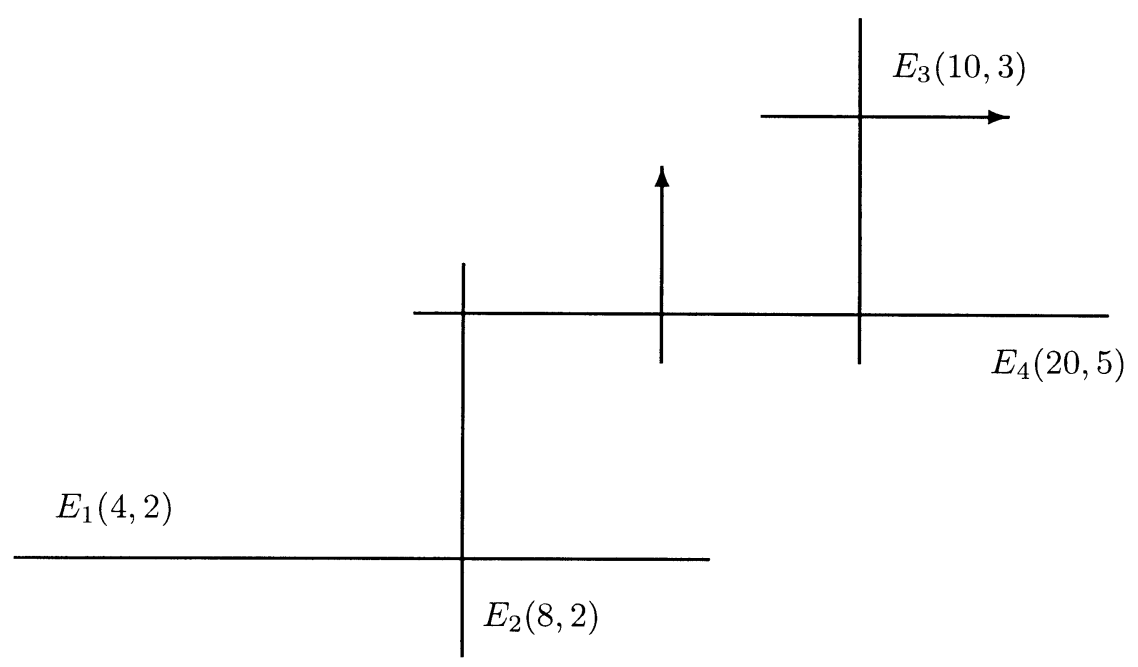

Figure 1.

In our case the resolution of the curve singularity $\left\{\left(z^{3}+y^{5}\right)^{2}=0\right\} \cap\{x=0\}$ is shown on the Figure 1. The numbers in brackets are the multiplicities of the corresponding components of the exceptional divisor in the zero divisor of the lifting of the germs $\left(z^{3}+y^{5}\right)^{2}$ and $x$ respectively.

Thus we have

$$
\zeta_{\varphi_{1}}=\left(1-t^{34}\right)^{-1}\left(1-t^{170}\right)
$$

Finally

$$
\zeta_{f}(t)=\frac{\left(1-t^{10}\right)\left(1+t^{11}\right)^{50}\left(1-t^{11}\right)\left(1-t^{330}\right)\left(1-t^{170}\right)}{\left(1-t^{66}\right)\left(1-t^{110}\right)\left(1-t^{34}\right)}
$$

3.5. Let $n+1$ be equal to 3 again, $f=f_{d}+f_{d+k}+\ldots, \mathcal{Z}\left(f_{d}\right)$ is a curve in $\mathbb{C P}^{2}$. Let $C_{1}^{q_{1}}+\ldots+C_{r}^{q_{r}}$ be its decomposition into irreducible components. Let $S\left(\mathcal{Z}_{\text {red }}\left(f_{d}\right)\right)$ consist of $s$ points $\left\{P_{1}, \ldots, P_{s}\right\}$. Suppose that:

(1) the curve $\mathcal{Z}\left(f_{d+k}\right)$ is reduced;

(2) $S\left(\mathcal{Z}_{\text {red }}\left(f_{d}\right)\right) \cap \mathcal{Z}\left(f_{d+k}\right)=\emptyset$

(3) for each $i$ with $q_{i}>1$ the curves $C_{i}$ and $\mathcal{Z}\left(f_{d+k}\right)$ intersect transversally, i.e. the set $C_{i} \cap \mathcal{Z}\left(f_{d+k}\right)$ consists of $d_{i}(d+k)$ different points $\left(d_{i}=\operatorname{deg} C_{i}\right)$.

In this case, the third author ([5]) has proved that the singularity $f$ has an isolated critical point at the origin it is $(d+k)$-determined and its Milnor number 
is equal to

$$
(d-1)^{3}+k \sum_{P_{i} \in S\left(\mathcal{Z}_{\text {red }}\left(f_{d}\right)\right)} \mu_{P_{i}}\left(\mathcal{Z}_{\text {red }}\left(f_{d}\right)\right)+k(d-\tilde{d})(2 d-3+\tilde{d})+k^{2}(d-\tilde{d}),
$$

where $\tilde{d}=d_{1}+\ldots+d_{r}$ is the degree of $\mathcal{Z}_{\text {red }}\left(f_{d}\right), \mu_{P_{i}}\left(\mathcal{Z}_{\text {red }}\left(f_{d}\right)\right)$ is the Milnor number of the (reduced) curve $\mathcal{Z}_{\text {red }}\left(f_{d}\right)$ at the point $P_{i}$.

Let $\pi:\left(\mathcal{X}, \mathbb{C P}^{2}\right) \rightarrow\left(\mathbb{C}^{3}, 0\right)$ be the blowing-up of the origin in $\left(\mathbb{C}^{3}, 0\right), \varphi$ the lifting of $f$ to the space $\mathcal{X}$. Let us consider the partitioning of the exceptional divisor $\mathbb{C P}^{2}$ into the following strata:

(a) 0-dimensional stratum $\Xi_{i}^{0}$ consisting of one point $P_{i}$ each $(i=1, \ldots, s)$;

(b) 0-dimensional stratum $\Lambda_{i}^{0}=C_{i} \cap \mathcal{Z}\left(f_{d+k}\right)$ for each $i$ with $q_{i}>1$;

(c) 1-dimensional stratum $\Xi_{i}^{1}=C_{i} \backslash\left\{S\left(\mathcal{Z}_{\text {red }}\left(f_{d}\right)\right) \cup M_{i}^{0}\right\}$ for each $i=1, \ldots, r$;

(d) 2-dimensional stratum $\Xi^{2}=\mathbb{C P}^{2} \backslash \mathcal{Z}_{\text {red }}\left(f_{d}\right)$.

At each point of $\Xi^{2}$, the lifting $\varphi$ of the germ $f$ has the normal form $u^{d}$. Its zeta-function is equal to $\left(1-t^{d}\right)$. The Euler characteristic of $\Xi^{2}$ is equal to

$$
\chi\left(\mathbb{C P}^{2}\right)-\chi\left(\mathcal{Z}\left(f_{d}\right)\right)=3-\chi(2, \tilde{d})+\sum_{i=1}^{s} \mu_{P_{i}}\left(\mathcal{Z}_{\text {red }}\left(f_{d}\right)\right) .
$$

At each point of $\Xi_{i}^{1}$, the lifting $\varphi$ has the form $u^{d}\left(y_{1}^{q_{i}}+u^{k}\right)$. Its zeta-function is equal to $\left(1-t^{\left(q_{i} / q_{i}^{\prime}\right)(d+k)}\right)^{-q_{i}^{\prime}}$, where $q_{i}^{\prime}=$ g.c.d. $\left(q_{i}, k\right)$. The Euler characteristic of $\Xi_{i}^{1}$ is equal to

$$
\chi\left(C_{i}\right)-d_{i}(d+k)-\sharp\left\{C_{i} \cap S\left(\mathcal{Z}_{\text {red }}\left(f_{d}\right)\right)\right\} .
$$

At each point of the stratum $\Lambda_{i}^{0}$, the lifting $\varphi$ has the normal form $u^{d}\left(y_{1}^{q_{i}}+u^{k} y_{2}\right)$. The zeta-function of it is equal to 1 .

At a point $P_{i}$, the lifting $\varphi$ has the form $u^{d}\left(g_{i}\left(y_{1}, y_{2}\right)+u^{k}\right)$, where $\left\{g_{i}=0\right\}$ is the local equation of the (non-reduced) curve $\mathcal{Z}\left(f_{d}\right)$ at the point $P_{i}$. The zeta-function of it is equal to

$$
\left(1-t^{d+k}\right)\left(\zeta_{g_{i}}^{k}\left(t^{d+k}\right)\right)^{-1}
$$

Remark 4. Again we can not apply Lemma 1 directly since the singularity of the germ $g$ is not isolated. However, it isn't difficult to see that, actually, the proof of Lemma 1 uses only the fact that the singularity of $g$ can be resolved by a modification which is an isomorphism outside the origin. This is so for curve singularities.

Thus we have

$$
\begin{aligned}
\zeta_{f}(t) & =\left(1-t^{d}\right)^{\chi\left(\Xi^{2}\right)} \prod_{i=1}^{r}\left[\left(1-t^{d+k}\right)\left(1-t^{\left(q_{i} / q_{i}^{\prime}\right)(d+k)}\right)^{-q_{i}^{\prime}}\right]^{\chi\left(\Xi_{i}^{1}\right)} . \\
& \cdot\left(1-t^{d+k}\right)^{s} \prod_{i=1}^{s}\left(\zeta_{g_{i}}^{k}\left(t^{d+k}\right)\right)^{-1} .
\end{aligned}
$$




\section{References}

[1] N. A'Campo, La fonction zeta d'une monodromie, Commentarii Mathematici Helvetici 50 (1975), 233-248.

[2] V. I. Arnold, S. M. Gusein-Zade, A. N. Varchenko, Singularities of Differentiable Maps, Vol. II, Birkhäuser, Boston, Basel, Berlin 1988.

[3] E. Artal-Bartolo, Forme de Jordan de la Monodromie des Singularités Superisolées de Surfaces, Memoirs of the A.M.S., Vol. 109, 525, 1994.

[4] I. Luengo, The $\mu$-constant stratum is not smooth, Inventiones mathematicae 90 (1987), 139-152.

[5] A. Melle-Hernández, Topología de singularidades de hipersuperficies: invariantes polares y número de Milnor, Ph.D. Thesis, Universidad Complutense de Madrid, 1996.

[6] D. Siersma, The monodromy of a series of hypersurfaces singularities, Commentarii Mathematici Helvetici 65 (1990), 181-197.

[7] J. Stevens, On the $\mu$-constant stratum and the V-filtration: an example, Mathematische Zeitschrift 201 (1989), 139-144.

[8] M. Tibăr, Lefschetz number of the monodromy and resolution of singularities, Indag. Mathem. N.S. 5 (1994), 119-128.

[9] A. N. Varchenko, Zeta function of monodromy and Newton's diagram, Inventiones mathematicae, 37 (1976), 253-262.

Sabir M. Gusein-Zade

Independent University of Moscow RUSSIA

e-mail: sabir@ium.ips.ras.ru

Ignacio Luengo

Departamento de Algebra

Facultad de Ciencias Matemáticas

Universidad Complutense de Madrid

Ciudad Universitaria

E-28040 Madrid

Spain

e-mail: iluengo@eucmos.sim.ucm.es

(Received: March 25, 1996)
Alejandro Melle-Hernández

Departamento de Algebra

Facultad de Ciencias Matemáticas

Universidad Complutense de Madrid

Ciudad Universitaria

E-28040 Madrid

Spain

e-mail: amelle@eucmos.sim.ucm.es 